8 Mayo Clinic. haplo.stats. R package version 1.3.1. http:// mayoresearch.mayo.edu/mayo/research/schaid_lab / software.cfm Date last accessed: October 3, 2008. Date last updated: April, 2008.

9 R Development Core Team. R Foundation for Statistical Computing. www.R-project.org Date last accessed: October 3, 2008. Date last updated: April, 2008.
10 Hassett C, Aicher L, Sidhu JS, Omiecinski CJ. Human microsomal epoxide hydrolase: genetic polymorphism and functional expression in vitro of amino acid variants. Hum Mol Genet 1994; 3: 421-428.

\title{
Pulmonary hypertension therapy and COPD: still many questions to be answered
}

\section{To the Editors:}

After great development in the knowledge of pulmonary arterial hypertension $(\mathrm{PAH})$ during the last decades, there is now an increasing interest in the pathophysiology of other forms of pulmonary hypertension, such as chronic obstructive pulmonary disease (COPD). The results obtained in the study by STOLZ et al. [1] have shown that for an average COPD Global Initiative for Chronic Obstructive Lung Disease stage III-IV population, the use of bosentan is not related to a significant improvement in exercise capacity and is associated with worsening of ventilation-perfusion mismatch. Although it is the first randomised study to address the use of bosentan, a proven PAH specific therapy, in the setting of COPD, the study design may lead to misinterpretation of the results. The study was not designed to treat COPD associated pulmonary hypertension. Furthermore, the absence of invasive measurements did not allow a proper subgroup analysis that could provide further support for the use of specific PAH therapy in the treatment of "disproportional" pulmonary hypertension associated with COPD. It is well known that most patients who present with pulmonary hypertension in the setting of COPD and other diseases actually have other associated causes for pulmonary hypertension development rather than an idiopathic PAH-like disease [2, 3], for instance, frequently presenting with some degree of left ventricle dysfunction. Since the study did not perform invasive haemodynamic assessment of the COPD patients, no assumption about a possible presence of post-capillary impairment, a situation in which bosentan has shown no effect so far [4], could be made.

Another matter of debate is the use of an echocardiogram to assess the presence of pulmonary hypertension in patients with severe COPD, since the technical difficulties in this specific clinical presentation have shown to impair the accuracy of this methodology.

In summary, we believe the authors have addressed an important question, but due to the chosen study design we remain with more questions than answers about the use of specific pulmonary arterial hypertension therapy in the setting of chronic obstructive pulmonary disease.

\section{Jardim and R. Souza}

Pulmonary Division, Heart Institute, University of São Paulo, São Paulo, Brazil.

\section{STATEMENT OF INTEREST}

None declared.

\section{REFERENCES}

1 Stolz D, Rasch H, Linka A, et al. A randomised, controlled trial of bosentan in severe COPD. Eur Respir J 2008; 32: 619-628.

2 Chaouat A, Bugnet AS, Kadaoui N, et al. Severe pulmonary hypertension and chronic obstructive pulmonary disease. Am J Respir Crit Care Med 2005; 172: 189-194.

3 Dias B, Lapa M, Figueiredo M, et al. Prevalence of pulmonary hypertension in hepatosplenic schistosomiasis. Am J Respir Crit Care Med 2008; 177: Suppl., A443.

4 Kaluski E, Cotter G, Leitman M, et al. Clinical and hemodynamic effects of bosentan dose optimization in symptomatic heart failure patients with severe systolic dysfunction, associated with secondary pulmonary hypertension-a multi-center randomized study. Cardiology 2008; 109: 273-280.

DOI: $10.1183 / 09031936.00146008$

\section{To the Editors:}

In the paper by STOLz et al. [1], bosentan deteriorated not only exercise capacity but also hypoxaemia and quality of life among subjects with severe or very severe chronic obstructive pulmonary disease (COPD). While the concept of a new drug category which could improve functional status among these patients is challenging, the theoretical background and clinical data to support the rationale for this trial [1] is rather weak.

In the study by GUNTHER et al. [2], the use of bosentan in 12 subjects with idiopathic pulmonary fibrosis was generally well 